\title{
Symmetry Switching of Negative Thermal Expansion by Chemical Control
}

\author{
Mark S. Senn, ${ }^{* \dagger}$ Claire A. Murray, ${ }^{\ddagger}$ Xuan Luo, ${ }^{\S}$ Lihai Wang, ${ }^{\S}$ Fei-Ting Huang, $"$ Sang-Wook Cheong, ${ }^{\S} \|$ \\ Alessandro Bombardi, ${ }^{\ddagger}$ Chris Ablitt, ${ }^{\perp}$ Arash A. Mostofi, ${ }^{\perp}$ and Nicholas C. Bristowe ${ }^{\perp^{\perp}}$ \\ ${ }^{\dagger}$ Department of Chemistry, University of Oxford, Inorganic Chemistry Laboratory, South Parks Road, Oxford, OX1 3QR, U.K. \\ ${ }^{\ddagger}$ Diamond Light Source, Harwell Campus, Oxfordshire, OX11 0DE, U.K. \\ ${ }^{\S}$ Laboratory for Pohang Emergent Materials and Max Plank POSTECH Center for Complex Phase Materials, Pohang University of \\ Science and Technology, Pohang 790-784, Korea \\ "Rutgers Center for Emergent Materials and Department of Physics Astronomy, Rutgers University, Piscataway, New Jersey 08854, \\ United States \\ ${ }^{\perp}$ Department of Materials, and the Thomas Young Centre, Imperial College London, London SW7 2AZ, U.K.
}

\section{Supporting Information}

ABSTRACT: The layered perovskite $\mathrm{Ca}_{3-x} \mathrm{Sr}_{x} \mathrm{Mn}_{2} \mathrm{O}_{7}$ is shown to exhibit a switching from a material exhibiting uniaxial negative to positive thermal expansion as a function of $x$. The switching is shown to be related to two closely competing phases with different symmetries. The negative thermal expansion (NTE) effect is maximized when the solid solution is tuned closest to this region of phase space but is switched off suddenly on passing though the transition. Our results show for the first time that, by understanding the symmetry of the competing phases alone, one may achieve unprecedented chemical control of this unusual property.

$\mathrm{E}$ ngineering a ground state structure to produce a desired physical property is becoming more widespread, particularly in the field of ferroelectrics where the symmetry of the paraelectric parent phase may be intentionally broken by chemical design leading to a polar distortion, e.g., ref 1 . However, many physical properties do not rely solely on the ground state structure, but are a result of dynamics and the phonon density of states; one such property is thermal expansion. Here we demonstrate that, by using chemical control, we can engineer competing crystallographic phases (with distinct symmetries) in the layered perovskite $\mathrm{Ca}_{3-x} \mathrm{Sr}_{x} \mathrm{Mn}_{2} \mathrm{O}_{7}$ that allow us to selectively switch on and off uniaxial negative thermal expansion (NTE). We show how this NTE arises from a soft phonon mode associated with this phase competition and how the property is enhanced at the point nearest to the transition. This precise tuning of NTE via chemical control of the crystallographic state suggests that tuning the symmetry of closely competing ground states in the wider perovskite family could be used to enhance and control this useful property.

Prediction of negative thermal expansion materials remains challenging. Identification of soft rigid unit modes (RUMs), ${ }^{2}$ and their success in explaining phase transitions and volume anomalies in framework silicates, ${ }^{3}$ has led to RUMs being used to understand thermal expansion in zirconium tungstate
$\left(\mathrm{ZrW}_{2} \mathrm{O}_{8}\right)$, ${ }^{4,5}$ often cited as the eponymous example of a NTE material. ${ }^{6}$ However, other systems exist such as $\mathrm{Cu}_{2} \mathrm{O}$ where a RUM description of the NTE has been shown to be inconsistent with experimental ${ }^{7}$ and theoretical ${ }^{8}$ observations and in $\mathrm{ScF}_{3}{ }^{9}$ where the quasi-harmonic approximation (implicit in any RUM formalism) has been shown to be insufficient. ${ }^{10}$ Furthermore, geometric arguments for designing materials exhibiting NTE due to RUMs ${ }^{11}$ provide at best a necessary (but not sufficient) criterion when searching for novel NTE materials. It is for example very well-known that the perovskite structure $\mathrm{ABO}_{3}$ has a large number of RUMs that could be expected to give rise to NTE; however, no reported examples exist to date. The reason for this is likely twofold. First, thermal expansion arises as a collective phenomenon, as it is due to the density of states weighted average of the Grüneisen parameters $^{12}$ of all phonon modes. Hence, it is not enough to identify a soft phonon mode whose transverse vibrations could lead to NTE, but one must also identify all contributions to thermal expansion from all phonon modes for the relevant energy scale, which is determined by $k_{\mathrm{B}} T$. Second, and most applicable to the perovskite systems, soft phonon mode phase transitions as a function of temperature are plentiful. ${ }^{13}$ The phonon modes that might give rise to NTE typically go soft and condense out over a temperature range of only a few kelvin, ${ }^{14}$ giving rise to displacive phase transitions, ${ }^{15}$ after which point they no longer contribute. While the first issue affects all predictions of NTE and may not be ignored if a quantitative understanding of NTE is sought, the design of systems with an active soft phonon mode over a large temperature range will undoubtedly lead to interesting bulk thermal expansion properties, especially at lower temperatures. Here, we demonstrate for a relatively simple layered perovskite system, which exhibits two competing ground states that may be precisely controlled by chemical composition, that uniaxial NTE may be switched on and off by manipulating the soft modes responsible for the phase transitions. We show how symmetry analysis of the competing phases reveals the

Received: December 17, 2015

Published: February 29, 2016 
underlying dynamics responsible for the NTE and how this may be optimized nearest to the phase boundary to enhance NTE.

Our model system is that of $\mathrm{Ca}_{3} \mathrm{Mn}_{2} \mathrm{O}_{7}$ which has been the subject of a theoretical prediction of a new kind of "hybrid" improper ferroelectricity ${ }^{17,18}$ and where we have recently reported the serendipitous discovery of uniaxial NTE in a competing crystallographic phase. ${ }^{19}$ The ferroelectric phase crystallizing in space group $A 2_{1} a m$ and the NTE phase in Acaa were found to exhibit a pronounced phase coexistence over a large temperature range. Symmetry analysis (Figure 1) reveals

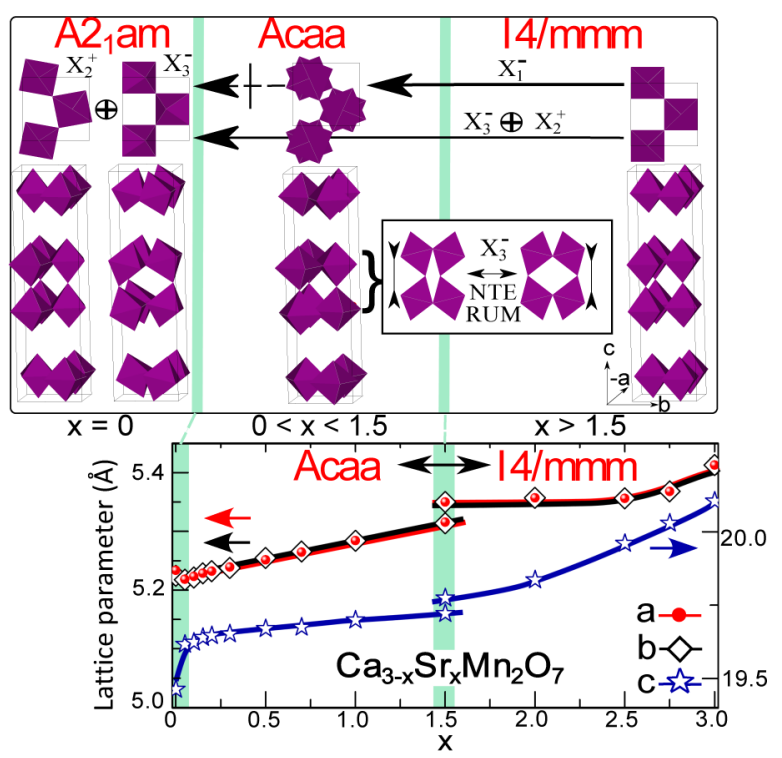

Figure 1. Scheme for chemical control of the symmetry of the solid solution $\mathrm{Ca}_{3-x} \mathrm{Sr}_{x} \mathrm{Mn}_{2} \mathrm{O}_{7}$ (top) with quantitative phase diagram based on ambient lattice parameters extracted from laboratory X-ray diffraction data (below). Symmetry allowed/forbidden phase transitions are indicated by solid arrows/broken lines. $14 / \mathrm{mmm}$ parent phase has unit cell $a=b \approx 3.8 \AA, c \approx 19.6 \AA$ with Ca1, Ca2, Mn1, O1, $\mathrm{O} 2, \mathrm{O} 3$ on $4 b, 8 e, 8 e, 4 a, 8 e$ and $16 i$ Wyckoff sites, respectively. $A 2_{1} a m$ has basis $[(1,-1,0),(-1,-1,0),(0,0,-1)]+\left(\frac{1}{4}, \frac{1}{4}, \frac{-1}{2}\right)$ and Acaa basis $[(1,-1,0),(1,1,0),(0,0,1)]+\left(0, \frac{1}{2}, 0\right)$ with respect to the parent setting. Irreducible representation labels follow ISODISTORT. ${ }^{16}$ The inset shows a RUM of $\mathrm{X}_{3}^{-}$character which we propose as being responsible for the NTE in the Acaa phase.

that there is no group-subgroup relationship between these phases meaning that the phase transition between them cannot be of second-order type. Decomposing these (child) structures in terms of symmetry adapted displacements of a hypothetical aristotypical structure (hereafter the parent structure) enables systematic classification of the ordered degrees of freedom in each phase. These ordered degrees of freedom may be thought of as phonon modes of the parent structure which have gone soft (frozen-in) in the displacive phase transitions producing the child structures. Hence, in favorable circumstances, the existence of a closely competing phase informs us about dynamics in the phase with which it is in competition with. The $A 2_{1} a m$ ground state phase has positive thermal expansion (PTE) along the $c$-axis and consists of a parent structure where two soft modes, one tilt and one rotation along the $c$-axis, have frozen in. The competing Acaa phase, which has NTE along the $c$-axis, only has a frozen-in rotational instability along the $c$-axis, leading us to speculate that it is the dynamical "left-over" degree of freedom in this phase (the tilt) which is responsible for the observed NTE. ${ }^{19}$ In Figure 1 we show how analysis of the irreducible representations of the competing structural distortions leads us to speculate that the NTE in the Acaa child phase is driven by the soft phonon mode of character $\mathrm{X}_{3}^{-}$(with respect to the parent phase) which may be visualized as a dynamic tilting of rigid $\mathrm{MnO}_{6}$ octahedral units about the $c$-axis (see inset of Figure 1).

To investigate the validity of this hypothesis we have prepared a polycrystalline sample of the solid solution $\mathrm{Ca}_{3-x} \mathrm{Sr}_{x} \mathrm{Mn}_{2} \mathrm{O}_{7}$ in the range $0 \leq x \leq 2$ via conventional solid state synthesis methods, with the aim of controlling not only the crystallographic symmetry observed at a particular temperature but also the proximity to a competing phase (Figure 1, lower panel). Figure 2 shows XRD diffraction

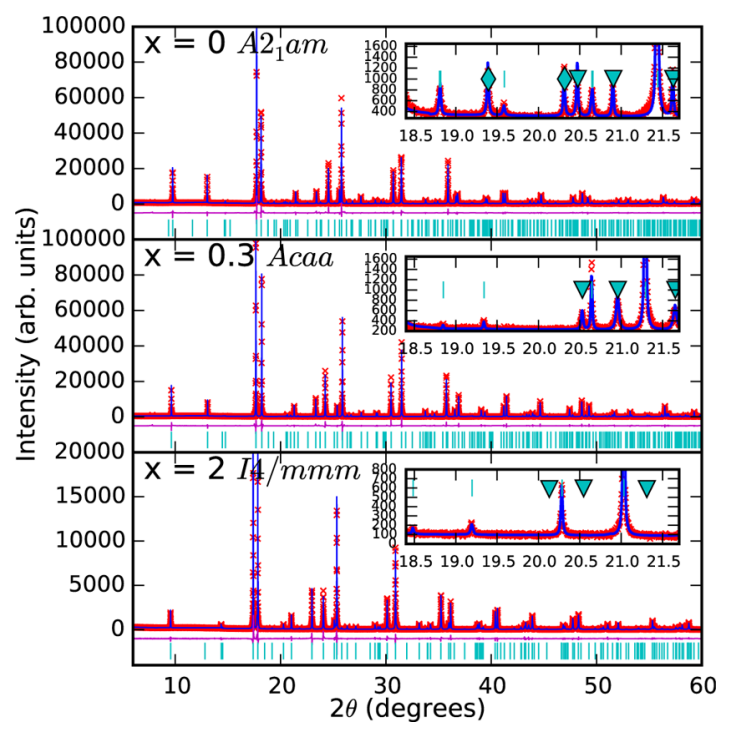

Figure 2. Rietveld refinement fits in the program Topas to SXRD data $(\lambda \approx 0.826 \AA)$ on the polycrystalline sample of $\mathrm{Ca}_{3-x} \mathrm{Sr}_{x} \mathrm{Mn}_{2} \mathrm{O}_{7}$ at $85 \mathrm{~K}$ for $x=0,0.3$, and 2 . Fits to the weak superstructure peaks evidence $A 2_{1} \mathrm{am}, A c a a$, and $14 / \mathrm{mmm}$ symmetries, respectively. Reflections allowed in the Acaa supercell but absent in $I 4 / \mathrm{mmm}$ are indicated by triangular symbols, and those additionally appearing in $A 2_{1} \mathrm{am}$ are indicated with diamonds.

patterns at $85 \mathrm{~K}$ which evidence space group symmetries of $A 2_{1} \mathrm{am}, A c a a$, and parent $I 4 / \mathrm{mmm}$ at $x=0,0.3$, and 2 respectively, as indicated by the Rietveld fit to the weak (super)structure peaks. Compositions $x=0.1$ and 0.5 are also found to crystallize in Acaa symmetry although the former exhibits a phase coexistence with $A 2_{1} a m(45 \%)$ when it is cooled to $85 \mathrm{~K}$ (see Supporting Information (SI)). Next we performed temperature-dependent XRD studies on the high resolution powder diffractometer I1 1 at Diamond Light Source, to accurately determine lattice parameters and thermal expansion coefficients. The evolution of the $\operatorname{strain}^{20}$ of the $c$ lattice parameter as a function of temperature is shown in Figure 3 for $x=0,0.1,0.2,0.3,0.5$, and 2. Volume and $a / b$ lattice parameters all display positive thermal expansion (PTE; see SI); however, the thermal expansion along $c$ shows an interesting switching behavior. Three regimes are evident at low temperature: at $x=0$, PTE is observed and the crystallographic symmetry is $A 2_{1} a m$; at $x=0.1-1.0$, NTE is observed and the crystallographic symmetry is Acaa (metrically pseudotetragonal within the resolution of our experiment); and finally, at $x=2$, 


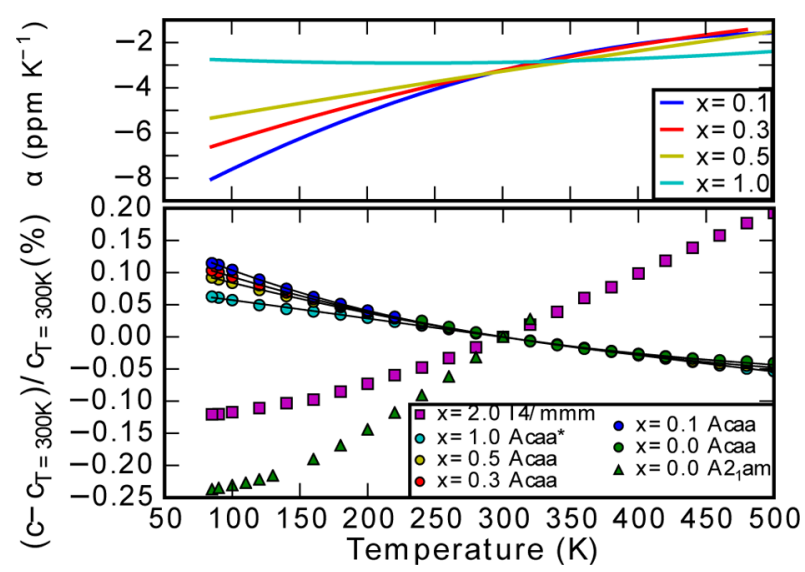

Figure 3. Evolution of strain (bottom) and $\alpha$ (top) for $c$ as a function of temperature for $\mathrm{Ca}_{3-x} \mathrm{Sr}_{x} \mathrm{Mn}_{2} \mathrm{O}_{7}$. Values for $x=0$ are calculated from ref 19. For $x=1$, unambiguous assignment of symmetry from the $\mathrm{XRD}$ was not possible and is instead made on the basis of Figure 1. $\alpha$ is calculated from an analytical derivative of a third-order polynomial fit to the strain.

PTE is observed and the space group is $I 4 / \mathrm{mmm}$. We have hence shown that uniaxial NTE may be suppressed through chemical control in two ways: first, by moving to a regime where the soft mode responsible for the NTE has frozen out and, hence, no longer contributes to the dynamics (the $A 2_{1} a m$ phase) and, second, by moving away from all structural instabilities such that none of the modes responsible for the phase transitions are close to being soft (i.e., the $14 / \mathrm{mmm}$ phase at $x=2$ ).

We now show that NTE is enhanced in the Acaa phase at the point in the solid solution of $x$ closest to the $A 2_{1} a m$ phase. In Figure 3 we plot the derivative of the strain, the thermal expansion coefficient $(\alpha)$. A strong negative correlation of $\alpha$ with $x$ is clearly evident. This enhancement of the NTE on approaching the competing phase $\left(A 2_{1} a m\right)$ and its immediate suppression on passing though the phase transition is indicative that the soft mode responsible for the phase transitions is also responsible for the observed NTE. Symmetry analysis alone of these three phases has revealed sufficient information on the dynamics to explain the observed NTE, something normally only possible after extensive theoretical work.

To further support the idea that the magnitude of the uniaxial NTE may be rationalized in terms of the proximity of the $A c a a$ and $A 2_{1} a m$ phases we have performed first-principles density-functional theory calculations on an analogous solid solution compound (see SI for details). At $0 \mathrm{~K}$ and ignoring zero-point energy contributions, we find $A 2_{1} a m$ to be lower in energy than Acaa for $x \leq 1.2$. At $x=1.2$ the energy difference between the two phases is $10 \mathrm{meV}$ per formula unit (see upper panel Figure 4). At higher values of $x, A 2_{1} a m$ ceases to be stable or metastable, whereas Acaa remains a metastable phase. Beyond $x=1.8$, Acaa is also no longer metastable, in good qualitative agreement with experiment. In a simplified picture, the low temperature NTE may be thought of as being dominated by the $\mathrm{X}_{3}^{-}$mode. Our calculations indeed show that the $\mathrm{X}_{3}^{-}$mode is the softest mode at $0 \mathrm{~K}$ in both the Acaa and $I 4 / \mathrm{mmm}$ phases $^{21}$ where it has not condensed out (see SI Tables 2 and 3). This mode is correlated with a reduction in the $c$ lattice parameter (see SI for details). Figure 4 plots the frequency of $\mathrm{X}_{3}^{-}$(in the $I 4 / \mathrm{mmm}$ phase for simplicity) as a function of $x$, showing that this mode becomes increasingly

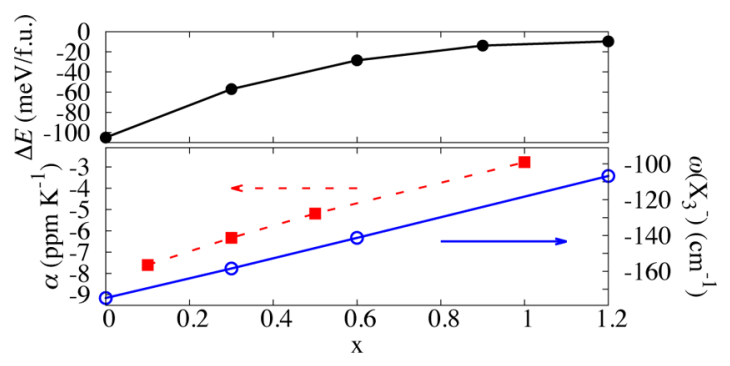

Figure 4. Energy difference between $A 2_{1} a m$ and Acaa phases (top) versus doping $x$. Experimental coefficient of thermal expansion $\alpha$ (at $100 \mathrm{~K})$ (bottom) and first-principles calculated frequency of the $\mathrm{X}_{3}{ }^{-}$ mode (in the $14 / \mathrm{mmm}$ phase), versus doping $x$. Negative frequencies indicate imaginary values (i.e., unstable modes).

unstable as $x$ is reduced. As shown in the same figure, this correlates with the measured low-temperature coefficient of thermal expansion, supporting the hypothesis that the $\mathrm{X}_{3}^{-}$mode is indeed responsible for NTE and that the magnitude of NTE can be related to the proximity to the $A 2_{1}$ am phase, in which the $\mathrm{X}_{3}^{-}$becomes soft and eventually condenses out. Despite their simplicity, these static calculations help us to understand and rationalize the experimentally observed trends, which suggests that design rules for optimizing NTE can be developed from an understanding of the symmetry alone.

Within the perovskite family there are a vast number of reported second-order phase transitions exhibiting structural distortions driven by soft modes with negative Grüneisen parameters. However, the fact that these modes go soft over a few kelvin ${ }^{14}$ results in only a short-lived anomaly in $\alpha . \mathrm{ScF}_{3}{ }^{9}$ and $\mathrm{ReO}_{3}{ }^{22}$ are the only reported examples of NTE in the extended structural family (A-site deficient perovskites), and here, proximity to a phase transition (in pressure) ${ }^{23,24}$ is likely indicative that these soft phonon modes dominate the thermal behavior. However, attempts to tune $\alpha$ by chemical substitution in $\mathrm{Sc}_{1-x} \mathrm{Y}_{x} \mathrm{~F}_{3}$ have been unsuccessful, resulting in phase transitions with a loss of the desired property. ${ }^{25}$ Very recently, tuning of NTE in $\mathrm{Sc}_{1-x} \mathrm{Al}_{x} \mathrm{~F}_{3}$ has shown some promise, ${ }^{26}$ but here the NTE is already suppressed (rather than enhanced) on approaching the cubic to rhombohedral phase transition from low to high $x$, suggesting that the anomalous thermal expansion behavior is not arising from the dominance of a single soft phonon mode (associated with the phase transition). Our system differs in that it is designed to have competing phases which have no group-subgroup relationship. This leads to a "trapping" of a soft mode over a large temperature range, and its dominance in the overall thermal expansion coefficient can clearly be seen at low temperatures. Our mechanistic understanding of NTE will be more widely applicable to a host of materials that are already reported to exhibit NTE, including a recent report of uniaxial NTE in $\mathrm{Sr}_{2} \mathrm{RhO}_{4}{ }^{27}$ We predict that the investigation of symmetry forbidden phase transitions in perovskite related compounds, and chemical control of these competing phases will provide a fruitful route for the design of materials exhibiting NTE and for tuning other physical properties related to the phonon density of states.

\section{ASSOCIATED CONTENT}

\section{Supporting Information}

The Supporting Information is available free of charge on the ACS Publications website at DOI: 10.1021/jacs.5b13192.

Crystallographic information files (ZIP) 
Synthesis, Rietveld refinement and computational details and associated data (PDF)

\section{AUTHOR INFORMATION}

\section{Corresponding Author}

*mark.senn@chem.ox.ac.uk

\section{Notes}

The authors declare no competing financial interest.

\section{ACKNOWLEDGMENTS}

We acknowledge Diamond Light Source for access to I11. Work at Rutgers University was supported by the DOE Grant No. DE-359 FG02-07ER46382, and that at Postech, by the Max Planck POSTECH/KOREA Research Initiative Program [Grant No. 2011-0031558] through NRF of Korea funded by MSIP. M.S.S. and N.C.B. acknowledge the Royal Commission for the Exhibition of 1851 for a fellowship. N.C.B. is also supported by a Junior Research Fellowship at Imperial College London. C.A. was supported through a studentship in the Centre for Doctoral Training on Theory and Simulation of Materials at Imperial College London funded by the EPSRC (EP/L015579/1). Calculations were performed on the Imperial College London high-performance computing facility. The cover artwork was produced using Vesta, ver 3.2.1 (Momma, K.; F. Izumi, F. VESTA 3 for three-dimensional visualization of crystal, volumetric, and morphology data. J. Appl. Crystallogr. 2011, 44, 1272-1276).

\section{REFERENCES}

(1) Mulder, A. T.; Benedek, N. A.; Rondinelli, J. M.; Fennie, C. J. Adv. Funct. Mater. 2013, 23, 4810-4820.

(2) Giddy, A. P.; Dove, M. T.; Pawley, G. S.; Heine, V. Acta Crystallogr., Sect. A: Found. Crystallogr. 1993, 49, 697-703.

(3) Hammonds, K. D.; Dove, M. T.; Giddy, A. P.; Heine, V.; Winkler, B. Am. Mineral. 1996, 81, 1057-1079.

(4) Pryde, A. K. A.; Hammonds, K. D.; Dove, M. T.; Heine, V.; Gale, J. D.; Warren, M. C. J. J. Phys.: Condens. Matter 1996, 8, 10973-10982.

(5) Tucker, M.; Goodwin, A.; Dove, M.; Keen, D.; Wells, S.; Evans, J. Phys. Rev. Lett. 2005, 95, 255501.

(6) Evans, J. S. O.; Mary, T. A.; Vogt, T.; Subramanian, M. A.; Sleight, A. W. Chem. Mater. 1996, 8, 2809-2823.

(7) Sanson, A.; Rocca, F.; Dalba, G.; Fornasini, P.; Grisenti, R.; Dapiaggi, M.; Artioli, G. Phys. Rev. B: Condens. Matter Mater. Phys. 2006, 73,214305 .

(8) Rimmer, L. H. N.; Dove, M. T.; Winkler, B.; Wilson, D. J.; Refson, K.; Goodwin, A. L. Phys. Rev. B: Condens. Matter Mater. Phys. 2014, 89, 214115.

(9) Greve, B. K.; Martin, K. L.; Lee, P. L.; Chupas, P. J.; Chapman, K. W.; Wilkinson, A. P. J. Am. Chem. Soc. 2010, 132, 15496-8.

(10) Li, C. W.; Tang, X.; Muñoz, J. A.; Keith, J. B.; Tracy, S. J.; Abernathy, D. L.; Fultz, B. Phys. Rev. Lett. 2011, 107, 195504.

(11) Grima, J. N.; Bajada, M.; Scerri, S.; Attard, D.; Dudek, K. K.; Gatt, R. Proc. R. Soc. London, Ser. A 2015, 471, 20150188.

(12) $\gamma_{i}=\delta \ln \left(\omega_{i}\right) / \delta \ln (V) ; \omega_{i}$ is the $i$ th mode frequency, and $V$, the volume.

(13) Cowley, R. a. Adv. Phys. 1980, 29, 1.

(14) Sollich, P.; Heine, V.; Dove, M. T. J. Phys.: Condens. Matter 1994, 6, 3171-3196.

(15) Dove, M. T. Am. Mineral. 1997, 82, 213-244.

(16) Campbell, B. J.; Stokes, H. T.; Tanner, D. E.; Hatch, D. M. J. Appl. Crystallogr. 2006, 39, 607-614.

(17) Benedek, N. A.; Fennie, C. J. Phys. Rev. Lett. 2011, 106, 107204.

(18) Harris, A. B. Phys. Rev. B: Condens. Matter Mater. Phys. 2011, 84, 064116.
(19) Senn, M. S.; Bombardi, A.; Murray, C. A.; Vecchini, C.; Scherillo, A.; Luo, X.; Cheong, S. W. Phys. Rev. Lett. 2015, 114, 35701.

(20) The strain is normalized at $T=300 \mathrm{~K}$ as the $x=0$ sample has a phase transition meaning that the lattice parameters can only be recovered for both symmetries at this point.

(21) At the X point $(0.5,0.5,0)$ with respect to the $I 4 / \mathrm{mmm}$ Brillouin zone.

(22) Chatterji, T.; Hansen, T. C.; Brunelli, M.; Henry, P. F. Appl. Phys. Lett. 2009, 94, 241902.

(23) Axe, J.; Fujii, Y.; Batlogg, B.; Greenblatt, M.; Di Gregorio, S. Phys. Rev. B: Condens. Matter Mater. Phys. 1985, 31, 663-66.

(24) Aleksandrov, K. S.; Voronov, V. N.; Vtyurin, A. N.; Krylov, A. S.; Molokeev, M. S.; Pavlovskiǐ, M. S.; Goryainov, S. V.; Likhacheva, A. Y.; Ancharov, A. I. Phys. Solid State 2009, 51, 810-816.

(25) Morelock, C. R.; Greve, B. K.; Gallington, L. C.; Chapman, K. W.; Wilkinson, A. P. J. Appl. Phys. 2013, 114, 213501.

(26) Morelock, C. R.; Gallington, L. C.; Wilkinson, A. P. J. Solid State Chem. 2015, 222, 96-102.

(27) Ranjbar, B.; Kennedy, B. J. Solid State Sci. 2015, 49, 43-46. 